\title{
Assessment of the Clinical and Procedural Predictive Factors of No-Reflow Phenomenon Following Primary Percutaneous Coronary Intervention
}

\author{
MAHMOUD M. SALEM, M.Sc.; HANAN K. KASEM, M.D.; MOHAMMAD E. EL-SETEHA, M.D. and \\ SUZAN B. EL-HEFNAWY, M.D.
}

The Department of Cardiovascular Medicine, Faculty of Medicine, Tanta University

\begin{abstract}
Background: Angiographic no-reflow phenomenon, a reduced coronary antegrade flow (TIMI flow grade <-2) without mechanical obstruction after recanalization, predicts poor LV functional recovery and survival in the early phase of STEMI. Although the predisposing factors of the no-reflow phenomenon were investigated, there is little data about clinical and procedural predictors of this phenomenon.
\end{abstract}

Aim of Study: The aim of this study was to evaluate the clinical and procedural predictive factors of no-reflow phenomenon following primary PCI.

Patients and Methods: The present study was conducted on 145 patients admitted with STEMI and treated with 1ry PCI at Cardiovascular Medicine Department, Tanta University Hospitals within 6 months from June 2016 to December 2016. Patients were divided into 2 groups according to no-reflow phenomenon. Group I: 29 patients with no reflow phenomenon. Group II: 116 patients without no reflow phenomenon. All patients were subjected to an informed consent, history taking including personal history, risk factors including Hypertension ( HTN), Diabetes Mellitus (DM), smoking, renal impairment. family history of premature coronary artery disease, past medical history of prior Myocardial Infarction (MI), Percutaneous Coronary Intervention (PCI) or Coronary Artery Bypass Graft (CABG), medications history, clinical examination including vital signs, Body Mass Index (BMI), signs of heart failure/hemodynamic instability according to Killip classification, signs of co-morbidities including renal/hepatic insufficiency, diabetes. Local cardiac examination, twelve leads surface ECG, echocardiography, blood sampling including serum cardiac biomarkers, complete blood count, lipid profile ( total cholesterol, HDL, LDL, triglycerides), random blood sugar on admission, serum urea \& creatinine on admission. Patients were subjected to diagnostic coronary angiography and primary PCI.

Results: The study demonstrated that there was a significant association between angiographic no-reflow and old

Correspondence to: Dr. Mahmoud M. Salem, The Department of Cardiovascular Medicine, Faculty of Medicine, Tanta University age, female gender, history of DM, prior MI, increased time to reperfusion, higher Killip class, decreased LV ejection fraction, increased blood CKMB, increased blood glucose, increased blood creatinine, the use of inotropes, initial TIMI flow grade 0 , high thrombus burden and stenting with ballon predilatation.

Conclusion: The occurrence of no-reflow phenomenon after primary PCI can be predicted using simple clinical, laboratory, angiographic and procedural features which include old age, female gender, history of DM, prior MI, increased time to reperfusion, higher Killip class, decreased LV ejection fraction, increased blood CKMB, increased blood glucose, increased blood creatinine, the use of inotropes, initial TIMI flow grade 0 , high thrombus burden and stenting with ballon predilatation.

Key Words: No-reflow phenomenon - Acute myocardial infarction - Percutaneous coronary intervention.

\section{Introduction}

CORONARY Artery Diseases (CAD) are the leading causes of morbidity and mortality in the developed countries. However the prognosis of Acute Myocardial Infarction (AMI) improved in the last decades due to the introduction of new pharmacological and mechanical reperfusion treatments allowing recanalization of the Infarct related artery ( IRA) $[1,2]$.

Primary Percutaneous Coronary Intervention ( PCI) is the gold standard of treatment of ST segment Elevation Myocardial Infarction (STEMI) [ 3]. The no-reflow phenomenon is defined as a profound reduction in antegrade coronary blood flow (TIMI flow grade <_2) despite vessel patency and the absence of dissection, spasm, or distal macroembolus [4-6]. It is presumed to reflect microvascular dysfunction [7,8]. Early detection, preventive measures and treatment of no reflow may alter the final outcome of PCI [9]. 
Aim of the study:

The aim of this study was to evaluate the clinical and procedural predictive factors of no-reflow phenomenon following primary PCI.

\section{Patients and Methods}

The present study was conducted on 145 patients admitted with STEMI and treated with 1ry PCI at the Cardiovascular Medicine Department, Tanta University Hospital from June 2016 to December 2016. Patients were divided into 2 groups according to no-reflow phenomenon. Group I: 29 patients with no reflow phenomenon. Group II: 116 patients without no reflow phenomenon.

Inclusion criteria were patients presenting with STEMI within 24 hours of symptoms and treated with primary PCI.

STEMI was defined as ST-segment elevation above $1 \mathrm{~mm}$ in at least two contiguous leads or new onset "or presumed new onset" LBBB combined with typical ischemic chest pain and/or elevated cardiac enzymes according to European Society of Cardiology (ESC) guidelines [10].

Exclusion criteria were patients presenting after 24 hours of symptoms and patients who received thrombolytic therapy.

All patients were subjected to history taking including personal history: Age, sex, risk factors including Hypertension (HTN), Diabetes Mellitus ( DM), smoking, renal impairment, family history of premature coronary artery disease (men under 55 years and women under 65 years), past medical history of prior MI, PCI or Coronary Artery Bypass Graft (CABG) and medications history.

Clinical examination including vital signs: e.g.: Heart rate, blood pressure, Body Mass Index (BMI), signs of heart failure, hemodynamic instability according to Killip classification, signs of comorbidities: Renal or hepatic insufficiency, diabetes, local cardiac examination, twelve leads surface Electrocardiogram (ECG), echocardiography including measurements, including ejection fraction, dimensions and segmental wall motion abnormalities. Blood sampling including serum cardiac biomarkers, complete blood count (Hemoglobin, hematocrite (Hct), total WBCs, neutrophils, lymphocytes, eosinophiles, basophiles, monocytes and platelets), lipid profile (total cholesterol, HDL, LDL, triglycerides), random blood sugar on admission, serum urea $\&$ creatinine on admission.
Patients were subjected to diagnostic coronary angiography and primary PCI with door to balloon time less than 90 minutes.

Thrombus aspiration, balloon pre-dilatation and post-dilatation were performed when indicated. The choice of stents (bare-metal stent or drugeluting stent) was left to the operator's discretion.

Reperfusion success is measured by TIMI blood flow grade: Reperfusion was considered successful ( TIMI 3) or abnormal (TIMI 0-1-2) according to the TIMI blood flow grade [11].

Statistical presentation and analysis of the present study was conducted, using the mean, standard deviation and chi-square test by SPSS V.20. Numerical data was presented as mean and Standard Deviation (SD) and categorical data was presented as number and percentage. Chi-squared test was used for statistical analysis. When the chisquared test was not appropriate, the likelihood ratio test was applied. The level of significance was adopted at $p<0.05$.

Subjects were informed about the purpose and procedure of the study and benefits of sharing in it. Ethical considerations of the study were carried out according to that of Declaration of Helsinki.

\section{Results}

Patients were divided into two groups, according to no-reflow phenomenon: Group (I): Patients with no reflow phenomenon $(n=29)$. Group (II): Patients without no reflow phenomenon $(n=116)$.

\section{Demographic data:}

Regarding the gender, Group I included 17 males (58.6\%) and 12 females (41.4\%), Group II included 90 males (77.6\%) and 26 females (22.4\%). There was statistically significant difference between the two groups as regarding the gender ( $p$ value $=0.038)($ Table 1$)$.

In group I, the age of the patients ranged from 49 to 75 years with a mean age of $60.21 \pm 6.74$ years. In group II the age ranged from 30 to 75 years with a mean age of $55.73 \pm 10.08$ years. There was statistically significant difference between the two groups as regarding the age ( $p$ value 0.006 )(Table 1 ).

\section{Risk factors:}

Risk factors of the study sample are shown in ( Table 2). There was a statistically significant 
Table (4): Comparison between the studied groups as regard to clinical characteristics.

\begin{tabular}{|c|c|c|c|c|}
\hline & $\begin{array}{l}\text { Group I } \\
(n=29)\end{array}$ & $\begin{array}{l}\text { Group II } \\
(n=116)\end{array}$ & Test of sig. & $p$ \\
\hline \multicolumn{5}{|c|}{ Time to reperfusion (hours): } \\
\hline Min.-max. & $2.0-22.0$ & $1.0-25.0$ & \multirow[t]{2}{*}{$\mathrm{U}=825.500 *$} & \multirow[t]{3}{*}{$<0.001 *$} \\
\hline Mean $\pm S D$ & $12.28 \pm 4.77$ & $8.29 \pm 4.74$ & & \\
\hline Median & 12.0 & 7.50 & & \\
\hline \multicolumn{5}{|l|}{ Systolic BP ( $\mathrm{mmHg})$ : } \\
\hline Min.-max. & $80.0-180.0$ & $60.0-210.0$ & \multirow[t]{2}{*}{$t=1.098$} & \multirow[t]{3}{*}{0.274} \\
\hline Mean $\pm S D$ & $123.79 \pm 24.23$ & $129.96 \pm 27.67$ & & \\
\hline Median & 120.0 & 130.0 & & \\
\hline \multicolumn{5}{|c|}{ Diastolic BP (mmHg): } \\
\hline Min.-max. & $50.0-120.0$ & $40.0-130.0$ & \multirow[t]{2}{*}{$t=0.558$} & \multirow[t]{3}{*}{0.578} \\
\hline Mean $\pm S D$ & $77.59 \pm 17.25$ & $79.31 \pm 14.26$ & & \\
\hline Median & 80.0 & 80.0 & & \\
\hline Heart rate $(\mathrm{b} / \mathrm{m})$ : & & & & \\
\hline Min.-max. & $49.0-130.0$ & $30.0-130.0$ & $t=0.107$ & 0.915 \\
\hline Mean $\pm S D$ & $87.90 \pm 22.44$ & $88.37 \pm 21.0$ & & \\
\hline Median & 90.0 & 90.0 & & \\
\hline Body mass index $(\mathrm{kg}$ & & & & \\
\hline Min.-max. & $21.0-31.0$ & $20.0-33.0$ & $t=0.217$ & 0.829 \\
\hline Mean $\pm S D$ & $25.41 \pm 2.78$ & $25.53 \pm 2.18$ & & \\
\hline Median & 25.0 & 25.0 & & \\
\hline Killip class: & No. & No. & & \\
\hline 1 & 72.4 & 88.8 & $\chi^{2}=5.025 *$ & $\mathrm{FE} p=0.037^{*}$ \\
\hline $2-4$ & 27.6 & 11.2 & & \\
\hline Infarction location: & No. & No. & & \\
\hline Anterior & 51.7 & 64.7 & $\chi^{2}=2.416$ & 0.274 \\
\hline Inferior & 48.3 & 32.8 & & \\
\hline Lateral & 0.0 & 2.6 & & \\
\hline Maximum ST elevat & & & & \\
\hline Min.-max. & $3.0-10.0$ & $1.0-12.0$ & $\mathrm{U}=1503.0$ & 0.368 \\
\hline Mean $\pm S D$ & $5.59 \pm 2.23$ & $4.88 \pm 2.02$ & & \\
\hline Median & 5.0 & 5.0 & & \\
\hline Number of $Q$ waves & & & & \\
\hline Min.-max. & $2.0-6.0$ & $0.0-7.0$ & $\mathrm{U}=1560.500$ & 0.535 \\
\hline Mean $\pm S D$ & $3.90 \pm 1.26$ & $3.62 \pm 1.40$ & & \\
\hline Median & 3.0 & 4.0 & & \\
\hline Ejection fraction (\% & & & & \\
\hline Min.-max. & $31.0-60.0$ & $30.0-70.0$ & $t=2.336^{*}$ & $0.025^{*}$ \\
\hline Mean $\pm S D$ & $45.45 \pm 9.26$ & $49.80 \pm 7.76$ & & \\
\hline Median & 45.0 & 50.0 & & \\
\hline
\end{tabular}

$\chi^{2}, p: \chi 2$ and $p$-values for Chi square test for comparing between the two groups.

$\mathrm{FE}_{p}: p$-value for Fisher Exact for Chi square test for comparing between the two groups.

$t, \quad p: t$ and $p$-values for Student $t$-test for comparing between the two groups.

$\mathrm{U}, p: \mathrm{U}$ and $p$-values for Mann Whitney test for comparing between the two groups. : Statistically significant at $p \leq 0.05$. 
Table (5): Comparison between the studied groups as regard to laboratory parameters on admission.

\begin{tabular}{|c|c|c|c|c|c|}
\hline Laboratory parameters & & $\begin{array}{l}\text { Group I } \\
(n=29)\end{array}$ & $\begin{array}{l}\text { Group II } \\
(n=116)\end{array}$ & Test of sig. & $p$ \\
\hline \multirow[t]{3}{*}{$C K M B(U / L)$} & Min.-max. & $73.0-250.0$ & $20.0-270.0$ & $\mathrm{U}=977.500 *$ & $<0.001 *$ \\
\hline & Mean $\pm S D$ & $14.66 \pm 37.93$ & $113.14 \pm 57.60$ & & \\
\hline & Median & 140.0 & 100.0 & & \\
\hline \multirow[t]{3}{*}{ Blood sugar at admission $(\mathrm{mg} / \mathrm{dl})$ : } & Min.-max. & $100.0-400.0$ & $80.0-600.0$ & $\mathrm{U}=1121.500^{*}$ & $0.006^{*}$ \\
\hline & Mean \pm SD & $225.31 \pm 79.48$ & $185.95 \pm 73.54$ & & \\
\hline & Median & 240.0 & 170.0 & & \\
\hline \multirow[t]{3}{*}{ Creatinine $(\mathrm{mg} / \mathrm{dl})$ : } & Min.-max. & $0.40-1.80$ & $0.60-2.30$ & $t=2.855^{*}$ & $0.005 *$ \\
\hline & Mean $\pm S D$ & $1.25 \pm 0.30$ & $1.09 \pm 0.26$ & & \\
\hline & Median & 1.20 & 1.0 & & \\
\hline \multirow[t]{3}{*}{ Total cholesterol ( $\mathrm{mg} / \mathrm{dl})$ : } & Min.-max. & $180.0-260.0$ & $143.0-320.0$ & $t=1.573$ & 0.118 \\
\hline & Mean \pm SD & $218.79 \pm 22.69$ & $209.0 \pm 30.74$ & & \\
\hline & Median & 220.0 & 210.0 & & \\
\hline \multirow[t]{3}{*}{$H D L(m g / d l):$} & Min.-max. & $33.0-45.0$ & $30.0-62.0$ & $t=0.203$ & 0.840 \\
\hline & Mean \pm SD & $41.0 \pm 3.55$ & $41.18 \pm 6.49$ & & \\
\hline & Median & 41.0 & 40.0 & & \\
\hline \multirow[t]{3}{*}{$L D L(m g / d l):$} & Min.-max. & $130.0-200.0$ & $82.0-250.0$ & $t=1.663$ & 0.100 \\
\hline & Mean \pm SD & $163.10 \pm 20.36$ & $154.45 \pm 38.52$ & & \\
\hline & Median & 169.0 & 148.0 & & \\
\hline \multirow[t]{3}{*}{ Triglycerides $(\mathrm{mg} / \mathrm{dl})$ : } & Min.-max. & $85.0-210.0$ & $53.0-300.0$ & $\mathrm{U}=1540.500$ & 0.484 \\
\hline & Mean \pm SD & $151.38 \pm 28.92$ & $153.16 \pm 58.28$ & & \\
\hline & Median & 155.0 & 140.0 & & \\
\hline \multirow[t]{3}{*}{ Hemoglobin $(g / d l)$ : } & Min.-max. & $10.0-16.0$ & $9.90-15.60$ & $t=0.940$ & 0.354 \\
\hline & Mean $\pm S D$ & $13.77 \pm 1.79$ & $13.44 \pm 1.05$ & & \\
\hline & Median & 14.0 & 13.50 & & \\
\hline \multirow[t]{3}{*}{$\operatorname{Hct}(\%)$ : } & Min.-max. & $35.0-48.0$ & $40.0-49.0$ & $\mathrm{U}=1580.500$ & 0.614 \\
\hline & Mean \pm SD & $40.38 \pm 3.13$ & $40.26 \pm 4.57$ & & \\
\hline & Median & 40.0 & 40.50 & & \\
\hline \multirow[t]{3}{*}{ Total WBCs $\left(X 10^{3} / \mathrm{mm}^{3}\right)$ : } & Min.-max. & $7.30-19.0$ & $5.50-33.0$ & $\mathrm{U}=1561.000$ & 0.550 \\
\hline & Mean \pm SD & $13.30 \pm 4.23$ & $12.57 \pm 3.91$ & & \\
\hline & Median & 14.0 & 11.50 & & \\
\hline \multirow[t]{3}{*}{ Neutrophils $\left(X 10^{3} / \mathrm{mm}^{3}\right)$ : } & Min.-max. & $5.80-16.0$ & $3.60-27.0$ & $\mathrm{U}=1523.000$ & 0.432 \\
\hline & Mean \pm SD & $10.01 \pm 3.23$ & $9.40 \pm 3.36$ & & \\
\hline & Median & 10.60 & 9.05 & & \\
\hline \multirow[t]{3}{*}{ Lymphocytes $\left(X 10^{3} / \mathrm{mm}^{3}\right)$ : } & Min.-max. & $0.90-5.30$ & $0.40-5.0$ & $\mathrm{U}=1614.0$ & 0.737 \\
\hline & Mean \pm SD & $2.55 \pm 1.40$ & $2.41 \pm 0.89$ & & \\
\hline & Median & 1.90 & 2.33 & & \\
\hline \multirow[t]{3}{*}{ Eosinophils $\left(/ \mathrm{mm}^{3}\right)$ : } & Min.-max. & $0.0-567.0$ & $0.0-1120.0$ & $\mathrm{U}=1645.500$ & 0.851 \\
\hline & Mean \pm SD & $165.21 \pm 171.69$ & $218.52 \pm 290.03$ & & \\
\hline & Median & 140.0 & 100.50 & & \\
\hline \multirow[t]{3}{*}{ Basophils $\left(/ \mathrm{mm}^{3}\right)$ : } & Min.-max. & $0.0-0.0$ & $0.0-200.0$ & $\mathrm{U}=16.38 .500$ & 0.383 \\
\hline & Mean \pm SD & $0.0 \pm 0.0$ & $3.19 \pm 21.61$ & & \\
\hline & Median & 0.0 & 0.0 & & \\
\hline \multirow[t]{3}{*}{ Monocytes $\left(/ \mathrm{mm}^{3}\right)$ : } & Min.-max. & $290.0-1600.0$ & $0.0-1870.0$ & $\mathrm{U}=1603.000$ & 0.696 \\
\hline & Mean \pm SD & $680.34 \pm 391.80$ & $684.44 \pm 373.35$ & & \\
\hline & Median & 600.0 & 670.0 & & \\
\hline \multirow[t]{3}{*}{ Platelets $\left(\times 10^{3} / \mathrm{mm}^{3}\right)$ : } & Min.-max. & $122.0-310.0$ & $108.0-410.0$ & $t=1.940$ & 0.054 \\
\hline & Mean \pm SD & $225.13 \pm 42.47$ & $246.98 \pm 56.73$ & & \\
\hline & Median & 220.0 & 240.0 & & \\
\hline
\end{tabular}

$t, \quad p: t$ and $p$-values for Student $t$-test for comparing between the two groups. $\mathrm{U}, p$ : $\mathrm{U}$ and $p$-values for Mann Whitney test for comparing between the two groups. : Statistically significant at $p \leq 0.05$. 
Table (6): Comparison between the studied groups as regard to angiographic characteristics.

\begin{tabular}{|c|c|c|c|c|c|c|c|}
\hline & & \multicolumn{2}{|r|}{$\begin{array}{l}\text { Group I } \\
(n=29)\end{array}$} & \multicolumn{2}{|c|}{$\begin{array}{l}\text { Group II } \\
(n=116)\end{array}$} & \multirow[t]{2}{*}{$x^{2}$} & \multirow[t]{2}{*}{$p$} \\
\hline & & No. & $\%$ & No. & $\%$ & & \\
\hline \multirow[t]{3}{*}{ Infarction related artery: } & LAD & 15 & 51.7 & 78 & 67.2 & 2.750 & $\mathrm{MC} p=0.223$ \\
\hline & $\mathrm{RCA}$ & 13 & 44.8 & 34 & 29.3 & & \\
\hline & LCX & 1 & 3.4 & 4 & 3.4 & & \\
\hline \multirow[t]{2}{*}{ Initial TIMI flow: } & 0 & 29 & 100.0 & 86 & 74.1 & $9.457 *$ & $0.002 *$ \\
\hline & $1-3$ & 0 & 0.0 & 30 & 25.9 & & \\
\hline \multirow[t]{2}{*}{ Number of diseased vessels: } & Single vessel & 12 & 41.4 & 60 & 51.7 & 0.993 & 0.319 \\
\hline & Multivessel & 17 & 58.6 & 56 & 48.3 & & \\
\hline \multirow[t]{2}{*}{ Thrombus burden: } & Low & 4 & 13.8 & 77 & 66.4 & $26.020 *$ & $<0.001 *$ \\
\hline & High & 25 & 86.2 & 39 & 33.6 & & \\
\hline \multirow[t]{4}{*}{ Target lesion location: } & Osteal & 9 & 31.0 & 18 & 15.5 & 3.907 & 0.142 \\
\hline & Proximal & 15 & 51.7 & 68 & 58.6 & & \\
\hline & Midsegment & 5 & 17.52 & 30 & 25.9 & & \\
\hline & Distal & 0 & 0.0 & 0 & 0.0 & & \\
\hline
\end{tabular}

M2 $\mathrm{P}: \chi^{2}$ and $p$-values for Chi square test for comparing between the two groups.

: $p$-value for Monte Carlo for Chi square test for comparing between the two groups.

: Statistically significant at $p \leq 0.05$.

Table (7): Comparison between the studied groups as regard to treatment options.

\begin{tabular}{|c|c|c|c|c|c|c|c|}
\hline & & \multicolumn{2}{|c|}{$\begin{array}{l}\text { Group I } \\
(\mathrm{n}=29)\end{array}$} & \multicolumn{2}{|c|}{$\begin{array}{l}\text { Group II } \\
(\mathrm{n}=116)\end{array}$} & \multirow[t]{2}{*}{$x^{2}$} & \multirow[t]{2}{*}{$p$} \\
\hline & & No. & $\%$ & No. & $\%$ & & \\
\hline \multirow[t]{2}{*}{ ADP receptor antagonist: } & Clopidogrel & 19 & 65.5 & 95 & 81.9 & 3.703 & 0.054 \\
\hline & Ticagrelor & 10 & 34.5 & 21 & 18.1 & & \\
\hline \multirow[t]{2}{*}{ Anticoagulation: } & UFH & 24 & 82.8 & 106 & 91.4 & 1.859 & $\mathrm{FE} p=0.182$ \\
\hline & LMWH & 5 & 17.2 & 10 & 8.6 & & \\
\hline \multirow[t]{2}{*}{ Inotropes: } & No & 22 & 75.9 & 106 & 91.4 & $5.398 *$ & ${ }^{\mathrm{FE}} p=0.046^{*}$ \\
\hline & Yes & 7 & 24.1 & 10 & 8.6 & & \\
\hline \multirow[t]{3}{*}{ Systemic glycoprotein IIb IIIa inhibitors: } & No & 0 & 0.0 & 2 & 1.7 & 5.777 & \\
\hline & Eptifibatide & 20 & 69.0 & 100 & 86.2 & & ${ }^{\mathrm{M}} \mathrm{C} p=0.057$ \\
\hline & Tirofeban & 9 & 31.0 & 14 & 12.1 & & \\
\hline \multirow[t]{3}{*}{ Type of intervention: } & Ballon angioplasty & 3 & 10.3 & 22 & 19.0 & $8.556^{*}$ & \\
\hline & Ballon + stenting & 22 & 75.9 & 53 & 45.7 & & $0.014 *$ \\
\hline & Direct stenting & 4 & 13.8 & 41 & 35.3 & & \\
\hline \multirow[t]{2}{*}{ Post stent dilatation: } & No & 20 & 69.0 & 98 & 84.5 & 3.686 & \\
\hline & Yes & 9 & 31.0 & 18 & 15.5 & & 0.055 \\
\hline \multirow[t]{3}{*}{ Number of stents: } & 0 & 3 & 10.3 & 22 & 19.0 & 5.134 & \\
\hline & 1 & 16 & 55.2 & 75 & 64.7 & & 0.077 \\
\hline & 2 & 10 & 34.5 & 16 & 16.4 & & \\
\hline \multirow[t]{3}{*}{ Type of stents: } & No stent & 3 & 10.3 & 22 & 19.0 & 1.511 & \\
\hline & BMS & 18 & 62.1 & 70 & 60.3 & & 0.470 \\
\hline & DES & 8 & 27.6 & 24 & 20.7 & & \\
\hline \multirow[t]{2}{*}{ Thrombus aspiration: } & No & 23 & 79.3 & 107 & 92.2 & 4.183 & \\
\hline & Yes & 6 & 20.7 & 9 & 7.8 & & $\mathrm{FE} p=0.080$ \\
\hline
\end{tabular}

$\mathrm{X} \sim^{2},: \chi^{2}$ and $p$-values for Chi square test for comparing between the two groups.

$p \mathrm{~F}: p$-value for Monte Carlo for Chi square test for comparing between the two

${ }^{1 v} \mathrm{E}_{p}$ groups. : $p$-value for Fisher Exact for Chi square test for comparing between the two groups. : Statistically significant at $p \leq 0.05$. 


\section{Discussion}

The rate of no-reflow phenomenon after primary PCI in the present study $(20 \%)$ was similar to that ( 12\%-25\%) reported previously in Piana et al. [12] and Morishima et al. [13].

In the current study, the predictors of angiographic no-reflow were old age, female gender, history of DM, prior MI, increased time to reperfusion, Killip class 2-4, decreased left ventricular ( LV) ejection fraction, increased blood CKMB, increased blood glucose, increased blood creatinine, the use of inotropes, initial TIMI flow grade 0 , high thrombus burden and stenting with ballon predilatation.

Sabin et al. [14] in their study which was conducted on 181 patients with STEMI who underwent primary PCI from August 2014 to February 2015, found that predictors of no reflow were age $>60$ years, reperfusion time $>6 \mathrm{~h}$, low initial TIMI flow ( 1), a high thrombus burden, a long target lesion, Killip Class III/IV and overlap stenting.

Abdi et al. [15] in their study which was conducted on 438 patients with STEMI who underwent primary PCI during an 18-month period, from 2013 to 2014 found that the predictive factors of the noreflow phenomenon in AMI patients undergoing primary PCI are: WBC count, thrombus grade, pain duration, maximal ST-changes, LV function, hs-CRP, bifurcation, eccentricity and coronary anatomy.

Celik et al. [16] demonstrated that female gender, pain to balloon time, high TIMI thrombus grade, tirofiban, mean platelet volume, and platelet lymphocyte ratio were independent predictors of no reflow after pPCI in young patients.

Kurtul et al. [17] investigated whether admission estimated glomerular filtration rate (eGFR) values are associated with no-reflow phenomenon in patients with STEMI treated with primary PCI. They reported that eGFR, Killip2 class, LV ejection fraction, and early patency of infarct vessel were independent predictors of no-reflow phenomenon.

Wang et al. [18] developed a simple scoring system to predict the risk of NRF in patients undergoing primary PCI with STEMI. The final model included 7 significant variables, which were age, pain-to-PCI time, neutrophil count, admission plasma glucose level, pre-PCI thrombus score, collateral circulation, and Killip class.
Kirma et al. [19] found that the occurrence of no-reflow phenomenon after primary PCI can be predicted using simple clinical, angiographic and procedural features which include advanced age ( $>60$ years), delayed reperfusion $\left(>\_4 h\right)$, low $(<1)$ TIMI flow prior to PCI, cut-off type total occlusion, high thrombus burden on baseline angiography, long target lesion $(>13.5 \mathrm{~mm})$ and large vessel diameter.

Ndrepepa et al. [20] reported that independent predictors of no reflow were residual flow in the infarct-related artery, initial perfusion defect, Creactive protein, and previous MI.

Dong-bao et al. [21] demonstrated that delayed reperfusion, high thrombus burden on baseline angiography, and blood glucose level on admission can be used to stratify AMI patients into a lower or higher risk for angiographic slow/no-reflow during PCI.

Zhou et al. [22] identified that age $>65$, long time from onset to reperfusion $>6$ hours, low SBP on admission $<100 \mathrm{mmHg}$, IABP use before PCI, low (" 1) TIMI flow grade before primary PCI, high thrombus burden, and long target lesion on angiography were independent predictors of no-reflow.

\section{Conclusion:}

The occurrence of no-reflow phenomenon after primary PCI can be predicted using simple clinical, laboratory, angiographic and procedural features which include old age, female gender, history of $\mathrm{DM}$, prior MI, increased time to reperfusion, higher Killip class, decreased LV ejection fraction, increased blood CKMB, increased blood glucose, increased blood creatinine, the use of inotropes, initial TIMI flow grade 0, high thrombus burden and stenting with ballon predilatation.

\section{Study limitations:}

The study had some limitations: First, this is a single-center experience and represents a limited number of patients. Second, the evaluation of noreflow was done by the TIMI flow grade only. As microvascular perfusion may also be reduced in patients with TIMI flow grade 3 , it would be better to be assessed by other angiographic measures like the TIMI frame count and the TIMI myocardial perfusion (TMP) grade (or myocardial blush grade).

\section{References}

1- FOX K.A., STEG P.G., EAGLE K.A., GOODMAN S.G., ANDERSON F.A., Jr., GRANGER C.B., et al.: Decline in rates of death and heart failure in acute coronary syndromes, 1999-2006. JAMA May 2; 297 (17): 1892900, 2007. 
2- DURANTE A. and CAMICI P.G.: Novel insights into an " old " phenomenon: The no reflow. Int J Cardiol., 187: 273-80, 2015.

3- O'GARA P.T., KUSHNER F.G., ASCHEIM D.D., CASEY D. E., Jr., CHUNG M.K., de LEMOS J.A., et al.: ACCF/ AHA guideline for the management of ST-elevation myocardial infarction: Executive summary: A report of the American College of Cardiology Foundation/American Heart Association Task Force on Practice Guidelines. J. Am. Coll. Cardiol. Jan., 29; 61 (4): 485-510, 2013.

4- REZKALLA S.H. and KLONER R.A.: Coronary Noreflow Phenomenon. Curr. Treat Options Cardiovasc. Med. May, 7 (1): 75-80, 2005.

5- EECKHOUT E. and KERN M.J.: The coronary no-reflow phenomenon: A review of mechanisms and therapies. Eur. Heart J. May, 22 (9): 729-39, 2001.

6- HENRIQUES J.P., ZIJLSTRA F., OTTERVANGER J.P., de BOER M.J., van't HOF A.W., HOORNTJE J.C., et al. Incidence and clinical significance of distal embolization during primary angioplasty for acute myocardial infarction. Eur. Heart J. Jul., 23 (14): 1112-7, 2002.

7- PRASAD A., STONE G.W., STUCKEY T.D., COSTANTINI C.O., ZIMETBAUM P.J., McLAUGHLIN M., et al.: Impact of diabetes mellitus on myocardial perfusion after primary angioplasty in patients with acute myocardial infarction. J. Am. Coll. Cardiol. Feb., 15; 45 (4): 508-14, 2005.

8- TIMMER J.R., van der HORST I.C., de L.G., OTTERVANGER J.P., HOORNTJE J.C., de BOER M.J., et al.: Comparison of myocardial perfusion after successful primary percutaneous coronary intervention in patients with ST-elevation myocardial infarction with versus without diabetes mellitus. Am. J. Cardiol. Jun., 1; 95 (11): 1375-7, 2005.

9- GUPTA S. and GUPTA M.M.: No reflow phenomenon in percutaneous coronary interventions in ST-segment elevation myocardial infarction. Indian Heart J. Jul., 68 ( 4): 539-51, 2016.

10- STEG P.G., JAMES S.K., ATAR D., BADANO L.P., BLOMSTROM-LUNDQVIST C., BORGER M.A., et al.: ESC Guidelines for the management of acute myocardial infarction in patients presenting with ST-segment elevation. Eur. Heart J. Oct., 33 (20): 2569-619, 2012.

11- MORROW D.A., ANTMAN E.M., CHARLESWORTH A., CAIRNS R., MURPHY S.A., de LEMOS J.A., et al.: TIMI risk score for ST-elevation myocardial infarction: A convenient, bedside, clinical score for risk assessment at presentation: An intravenous nPA for treatment of infarcting myocardium early II trial substudy. CirculationOct., 24; 102 (17): 2031-7, 2000.

12- PIANA R.N., PAIK G.Y., MOSCUCCI M., COHEN D.J., GIBSON C.M., KUGELMASS A.D., et al.: Incidence and treatment of 'no-reflow' after percutaneous coronary intervention. Circulation Jun., 89: 6.1-1111, 1994.
13- MORISHIMA I., SONE T., OKUMURA K., TSUBOI H., KONDO J., MUKAWA H., et al.: Angiographic no-reflow phenomenon as a predictor of adverse long-term outcome in patients treated with percutaneous transluminal coronary angioplasty for first acute myocardial infarction. J. Am.Coll. Cardiol. Oct., 36 (4): 1202-9, 2000.

14- SABIN P., KOSHY A.G., GUPTA P.N., SANJAI P.V., SIVAPRASAD K., VELAPPAN P., et al.: Predictors of no- reflow during primary angioplasty for acute myocardial infarction, from Medical College Hospital, Trivandrum. Indian Heart J. Apr., 69 (Suppl 1): S34-S45, 2017.

15- ABDI S., RAFIZADEH O., PEIGHAMBARI M., BASIRI H. and BAKHSHANDEH H.: Evaluation of the Clinical and Procedural Predictive Factors of no-Reflow Phenomenon Following Primary Percutaneous Coronary Intervention. Res. Cardiovasc. Med. May, 4 (2): e25414, 2015.

16- CELIK T., BALTA S., OZTURK C., KAYA M.G., APARCI M., YILDIRIM O.A., et al.: Predictors of No-Reflow Phenomenon in Young Patients With Acute ST-Segment Elevation Myocardial Infarction Undergoing Primary Percutaneous Coronary Intervention. Angiology Aug., 67 (7): 683-9, 2016 .

17- KURTUL A., MURAT S.N., YARLIOGLUES M., DURAN M., CELIK I.E. and KILIC A.: Mild to Moderate Renal Impairment Is Associated With No-Reflow Phenomenon After Primary Percutaneous Coronary Intervention in Acute Myocardial Infarction. Angiology Aug., 66 (7): 644-51, 2015.

18- WANG J.W., ZHOU Z.Q., CHEN Y.D., WANG C.H. and ZHU X.L.: A risk score for no reflow in patients with STsegment elevation myocardial infarction after primary percutaneous coronary intervention. Clin. Cardiol. Apr., 38 (4): 208-15, 2015.

19- KIRMA C., IZGI A., DUNDAR C., TANALP A.C., ODUNCU V., AUNG S.M., et al.: Clinical and procedural predictors of no-reflow phenomenon after primary percutaneous coronary interventions: Experience at a single center. Circ. J. May, 72 (5): 716-21, 2008.

20- NDREPEPA G., TIROCH K., KETA D., FUSARO M., SEYFARTH M., PACHE J., et al.: Predictive factors and impact of no reflow after primary percutaneous coronary intervention in patients with acute myocardial infarction. Circ. Cardiovasc. Interv. Feb., 1; 3 (1): 27-33, 2010.

21- DONG-BAO L., QI H., ZHI L., SHAN W. and WEI-YING $\mathrm{J} .:$ Predictors and long-term prognosis of angiographic slow/no-reflow phenomenon during emergency percutaneous coronary intervention for ST-elevated acute myocardial infarction. Clin. Cardiol. Dec., 33 (12): E7-12, 2010.

22- ZHOU H., HE X.Y., ZHUANG S.W., WANG J., LAI Y., QI W.G., et al.: Clinical and procedural predictors of noreflow in patients with acute myocardial infarction after primary percutaneous coronary intervention. World $\mathrm{J}$. Emerg. Med., 5 (2): 96-102, 2014. 


\section{تقييم العوامل الإكلينيكية والإجرائية التنبؤية

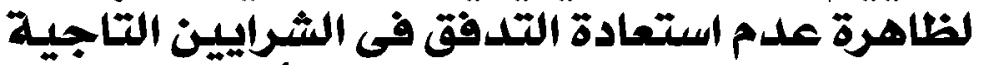

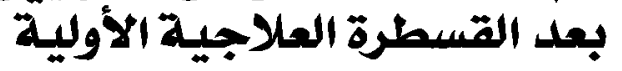

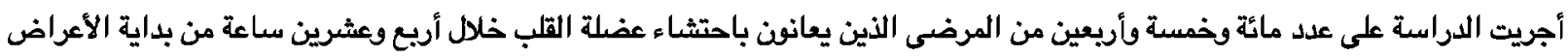

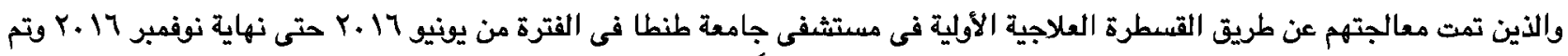

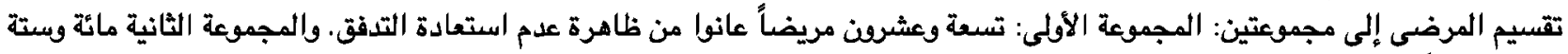

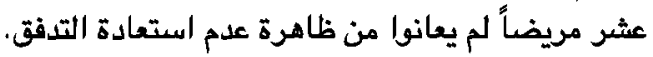

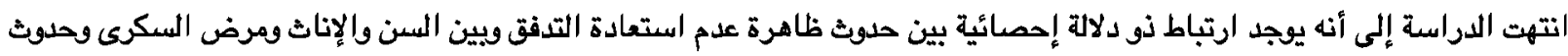

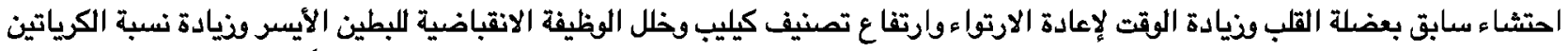

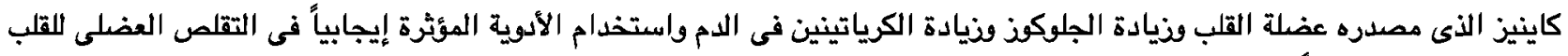

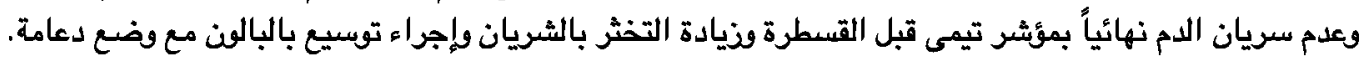

